\title{
Émergence des voix amérindiennes dans la littérature brésilienne
}

\section{Emergence of Amerindian voices in Brazilian literature}

\author{
Rita Olivieri-Godet ${ }^{1}$
}

Submetido em 10 de dezembro de 2016 e aprovado em 19 de janeiro de 2017.

Résumé: L'émergence des voix amérindiennes dans les lettres brésiliennes contribue à la reconfiguration de l'imaginaire sur les Amérindiens, tout en ouvrant un dialogue avec la tradition littéraire et les intertextes sociaux. Cet article s'intéresse aux raisons de leur émergence et insertion tardives dans le système littéraire brésilien en présentant, dans un premier temps, une vue d'ensemble du problème pour, dans un deuxième temps, se consacrer aux formes et thématiques prisées par la littérature amérindienne qui s'exprime en langue portugaise.

Mots-clefs: Émergence. Littérature amérindienne. Système littéraire brésilien.

Abstract: The emergence of Amerindian voices in the Brazilian literature contributes to some reconfiguration of the imaginary on the Amerindians, while opening a dialogue with literary tradition and social intertextuality. This article examines the reasons for their late emergence and insertion in the Brazilian literary system by presenting, at first, an overview of the problem and, secondly, by focusing on the valued forms and themes of the Amerindian literature in Portuguese language.

Keywords: Emergence. Amerindian literature. Brazilian literary system.

Ce travail s'intéresse au phénomène récent de l'émergence de la littérature amérindienne contemporaine de langue portugaise et à son insertion dans le marché éditorial des lettres brésiliennes.

Dans une première partie, je me consacrerai à présenter une première vue d'ensemble du problème. J'évoquerai d'abord la diversité des Peuples Premiers et celle de leurs productions intellectuelles en rapport avec les différentes phases d'interlocution avec la société nationale: récits oraux traditionnels, textes ethnographiques, récits mythiques, essais politiques, «auto-anthropologie» (VIVEIROS DE CASTRO, 2015, p. 11), littérature de jeunesse, textes romanesques et poétiques, production audiovisuelle et musicale. Il s'agira ensuite de rappeler le contexte du passage de l'oral à l'écrit et de l'appropriation de la langue portugaise qui a engendré de nouvelles formes et thématiques et a contribué à élargir l'insertion de ces productions dans le territoire culturel brésilien. 
L'examen de ce processus révèle notamment le rôle de l'écriture comme une tactique fondamentale à laquelle les Amérindiens ont recours pour établir un dialogue avec la culture hégémonique et s'affirmer en tant que sujet de leur propre histoire au sein d'une société nationale qui a tendance à les rejeter, les massacrer, les infantiliser, ou même à les rendre invisibles par l'effacement de leur histoire et de leur mémoire.

Dans une deuxième partie, je me consacrerai aux formes et thématiques de la littérature amérindienne dans le système littéraire brésilien tout en esquissant les principaux aspects des trajectoires sociales de ses écrivains. Je rappellerai brièvement les représentations des Amérindiens par des écrivains qui appartiennent au système traditionnel brésilien avant de me livrer à l'examen de l'irruption de la voix amérindienne dans ce système par le biais de la littérature de jeunesse, du roman et de la poésie. Ce phénomène fait de l'Amérindien non seulement le sujet privilégié des représentations mais aussi le sujet de l'énonciation dans les lettres brésiliennes. Pour examiner plus en détail l'élargissement et la reconfiguration du système littéraire traditionnel brésilien engendrés par l'inclusion de la voix amérindienne, je m'attacherai enfin à interroger la voix amérindienne féminine d'Eliane Potiguara dans son ouvrage Metade cara, metade máscara (2004).

\section{Diversité des Peuples Premiers et multiplicité d'expressions littéraires amérindiennes}

La diversité des peuples autochtones du Brésil ainsi que les singularités de leur parcours historique, notamment en ce qui concerne les contacts plus ou moins intenses avec la société brésilienne qui déterminent leur rapport à l'Etat et aux institutions, expliquent l'existence simultanée de plusieurs expressions littéraires amérindiennes. Victimes des politiques d'extermination ou d'intégration forcée, de nombreux peuples autochtones ont migré en dehors de leurs territoires traditionnels, ce qui a déclenché un processus plus ou moins développé de rapprochement du modèle occidental et d'insertion dans la société nationale. Comme le souligne Roland Walter, «dans les Amériques, la brutalisation des personnes est liée à la brutalisation de l'espace et ces brutalisations sont enracinées dans le passé»(WALTER, 2013, p. 9). Elles continuent néanmoins à avoir un impact et à se reproduire dans le présent. L'interaction inégale entre ces peuples 
amérindiens et la société nationale brésilienne a conduit à des formes ségrégationnistes d'appropriation sociale de l'espace. Si 57,7\% d'Amérindiens (THÉRY, 2014) vivent dans des territoires indigènes - à dimensions, conditions de survie et intensité de contact avec les «Blancs» très dissemblables -, d'autres (desaldeados) habitent à l'extérieur de ces territoires ayant quitté leurs villages traditionnels, pour s'installer dans des zones rurales, dans des campements au bord des routes, dans des quartiers pauvres des petites, moyennes et grandes villes brésiliennes. Depuis la colonisation, les peuples autochtones n'ont jamais été à l'abri de migrations forcées. De nos jours, nombreux sont les descendants de migrants Indiens dont la famille s'est vue obligée d'abandonner son territoire et ses formes d'organisation sociale pour aller vers les villes, d'adopter les pratiques et les coutumes de la société nationale, de perdre toutes références culturelles y compris, dans certains cas, la conscience même de son origine. D'autres migrants Indiens, tout en vivant en milieu urbain, redécouvrent et se réapproprient leur culture. Ce même phénomène se manifeste à l'intérieur des terres, où des Indiens «redécouverts» (Indios ressurgidos) qui ont pris conscience de leur identité luttent pour récupérer leurs territoires et faire revivre leurs cultures traditionnelles.

Dans le contexte actuel d'intensification des contacts entre les deux sociétés et de prise de conscience politique par les Peuples Premiers, on peut observer une transformation et un renouvellement des expressions artistiques amérindiennes au fur et à mesure que ces peuples s'emparent de l'écriture et des ressources techniques audiovisuelles pour faire entendre leur voix. La création artistique devient peu à peu une arme efficace pour s'immiscer dans le marché de la production artistique occidentale et faire bouger l'imaginaire sur les peuples et cultures autochtones. Les jeunes jouent un rôle prépondérant dans cet élan de mobilité artistique et deviennent acteurs de la production audiovisuelle, dont le projet Video nas aldeias est un exemple phare; de projets musicaux, à l'instar du groupe de rap $O z$ Guarani; de projets littéraires divers que nous analyserons dans la suite de ce travail. De plus en plus connectée, la jeunesse amérindienne se sert de réseaux médiatiques pour diffuser ses productions artistiques, qu'elles soient hybrides ou traditionnelles, et lutter contre l'exclusion matérielle et symbolique dont elle est victime. 


\section{L'oralité et l'écrit}

De nos jours, la tradition orale portée par une voix collective côtoie des textes portés par des voix collectives et individuelles. La littérature orale amérindienne au Brésil avec ses genres traditionnels (mythes, légendes, chants, etc.) poursuit son chemin dans les villages où les chamans et les anciens se chargent de sa transmission à de nouvelles générations. Les récits racontés et transmis par ces vraies archives vivantes du savoir ancestral trouvent de nouveaux supports matériels tels que l'enregistrement de la voix, la vidéo, l'écriture et l'édition.

Le phénomène culturel récent du passage des récits traditionnels de l'oral à l'écrit surgit au début des années 1990 et devient récurrent au XXI ${ }^{\mathrm{e}}$ siècle. La reconnaissance de l'existence des langues amérindiennes au Brésil (par la Constitution de 1988) a ouvert un chemin à l'éducation bilingue amérindienne et a contribué à la création de «l'école indigène» (SOUZA, 2004, p. 129). C'est ainsi qu'apparaissent des transcriptions de la tradition littéraire orale en langue minoritaire produites, comme le signale la spécialiste des textualités amérindiennes Maria Inês de Almeida, «dans le contexte de l'implantation de l'éducation scolaire dans les villages, c'est-à-dire qu'il s'agit d'une littérature financée par le gouvernement, dont la production/circulation est restreinte et n'entre pas dans le circuit commercial» (ALMEIDA, 2011). Il est important de souligner l'implication du milieu universitaire dont le projet Literaterras: escrita, leitura, traduções (Littératerres: écriture, lecture, traductions) coordonné par Maria Inês de Almeida à 1'Universidade Federal de Minas Gerais, constitue l'exemple pionnier d'un système d'éducation développé en interaction et avec la collaboration des étudiants/enseignants autochtones.

On constate également, l'existence de récits mythiques, ou qui présentent des enseignements à transmettre à d'autres générations, traduits en portugais, qui résultent d'une création collective par de jeunes auteurs amérindiens comme Antes o mundo não existia (des peuples Desana) e Shenipabu Miyui (des peuples Kaxinawá) entre autres. Comme le fait remarquer M. I. Almeida, les jeunes Indiens jouent un rôle fondamental dans la transposition à l'écrit, la traduction et l'édition des récits traditionnels, travail qu'ils assument au nom de leur peuple, tout en refusant la signature en tant qu'auteur individuel. 
Importants également sont les témoignages d'Amérindiens enregistrés, réécrits, traduits et publiés par des anthropologues ou des linguistes, dans le but d'établir un dialogue avec les représentants du monde occidental. L'ouvrage La chute du ciel. Paroles d'un chaman Yanomami en est un exemple, publié en France en 2010 et paru au Brésil en 2015 chez l'une des plus prestigieuses maisons d'édition, la Companhia das Letras. Il s'agit d'un vrai chef d'œuvre d'exercice de traversée culturelle issu d'une collaboration d'une dizaine d'années entre le chaman yanomami Davi Kopenawa, un des plus importants leaders du mouvement indien au Brésil, et Bruce Albert, anthropologue français. Dans le même domaine de l'approche interculturelle, il faut souligner le travail pionnier d'Yvete Walty, d'Ana L. Queiroz et de Leda L. Leonel qui ont recueilli et publié, en 1988, le témoignage de Pichuvy Cinta Larga sur l'histoire de son peuple: «après des siècles d'un monologue imposé à l'Indien - écrit Ivete Walty dans un article consacré aux rapports entre l'intellectuel et les minorités - le Non-Indien se propose d'être son interlocuteur» (WALTY, 2000).

D'autres ouvrages, issus de l'interlocution entre Indiens et Non-Indiens directement liés à l'organisation politique des Amérindiens, voient le jour dont celui d'Ailton Krenak, Encontros (2015). Cet ouvrage rassemble une série d'interviews de l'auteur, un des militants les plus renommés et actifs de la cause indienne au Brésil. Son témoignage fait surtout appel à l'histoire récente de la dépossession territoriale et des conflits qui en résultent, dénonçant les préjugés et les obstacles contre lesquels les peuples autochtones doivent se battre pour sortir de leur invisibilité, sans oublier le chemin parcouru dans le combat pour la conquête de leurs droits. L'engagement dans la lutte politique a propulsé Ailton Krenak au-devant de la scène nationale. Avec une trajectoire sociale distincte de celle de Davi Kopenawa qui habite les territoires yanomamis, Ailton Krenak est un Indien migrant, vivant à São Paulo où sa famille a migré en 1968 et l'a scolarisé. Il est né en 1953 dans une réserve créée en 1922, située dans la vallée de Rio Doce, dans le but, selon ses propres mots, d'emprisonner les Krenak dont les territoires traditionnels s'étendent du littoral de l'État de l'Espírito Santo au sertão du Minas Gerais.

Un chaman habitant la forêt amazonienne ; un journaliste enseignant à l'université, vivant dans la plus grande métropole de l'Amérique latine; deux parcours de vie 
militants; deux modes de production écrite insérés dans le circuit culturel des sociétés occidentales; deux trajectoires qui symbolisent la diversité de situations et de rapports que les Amérindiens entretiennent avec la société brésilienne; deux Amérindiens qui portent leur regard sur cette société, l'interprètent et la représentent. Dans leur discours, «l'autre sommes nous, les Blancs, des frères nés de la fertile et magique imagination indigène, plus généreuse avec nous que nous n'avons été avec eux» (SANTOS, 2003, p. 57). Eduardo Viveiros de Castro, célèbre anthropologue brésilien, attire l'attention sur le sens de leur parcours en tant qu'Indiens appartenant à une génération supposée être acculturée, mais qui en réalité «[...] se tornaram vetores de indigenização da política nacional, antes que de abrasileiramento dos índios, e que se reapropriaram de sua indianidade de modo simultaneamente intelectual e existencial $»^{2}$ (VIVEIROS DE CASTRO, 2015, p. 12). Une véritable prise de conscience politique des Amérindiens par l'écriture, qu'elle corresponde à des formes de discours issues de la tradition orale, comme chez Kopenawa, ou qu'elle s'adapte à un nouveau mode de communication, à l'instar d'Ailton Krenak.

\section{La figuration des Amérindiens dans la littérature brésilienne contemporaine}

Face à la répercussion des discours sociaux sur la question indienne au Brésil, la présence clairsemée de la création littéraire amérindienne écrite en portugais passe pratiquement inaperçue. Dans une étude consacrée à la figure de l'Amérindien dans des œuvres qui circulent dans le système littéraire traditionnel au Brésil, au Québec et en Argentine, j'ai pu constater que le système québécois était le seul à présenter un corpus conséquent d'ouvrages écrits par des auteurs qui revendiquent leur appartenance ethnique amérindienne. Dans l'ouvrage en question, L'altérité amérindienne dans la fiction des Amériques (OLIVIERI-GODET, 2015), je mettais en avant l'importance d'un ensemble de romans contemporains brésiliens, écrits par des non-Indiens, publiés depuis la deuxième moitié des années 1970 jusqu'à nos jours. Ces œuvres partent de la figuration de l'altérité amérindienne pour questionner les relations interculturelles et interethniques, en tenant compte de l'articulation entre la relecture du passé et le contexte historique actuel, dans lequel le processus de dépossession des Amérindiens de leurs terres apparaît comme un 
des éléments centraux. Ces écrivains n'envisagent pas de devenir des porte-paroles des Amérindiens, mais la parole qu'ils portent sur ces peuples dans leurs œuvres fictionnelles contribue à maintenir vivante la présence des Amérindiens dans l'espace symbolique de la représentation. Ils parlent d'un autre, de cet «étranger de dedans» que l'Amérindien représente aux yeux de la société brésilienne.

\section{Littérature amérindienne écrite en portugais dans le système littéraire brésilien}

Contrastant avec le poids de cette production insérée dans le système littéraire brésilien, des ouvrages fictionnels, poétiques ou dramaturgiques écrits par des Amérindiens sont inexistants dans le réseau littéraire brésilien avant la décennie de 1990. De nos jours encore, leur présence se fait toujours très discrète malgré un contexte apparemment favorable à l'impulsion d'une expression de la littérature amérindienne écrite en portugais, eu égard aux facteurs suivants: l'intérêt accru des discours sociaux pour les questions indiennes au Brésil; l'organisation et la consolidation des mouvements sociaux amérindiens, notamment à partir des années 198; 1'importance de la discussion autour des politiques identitaires minoritaires; l'accès d'une partie de la population amérindienne à l'éducation et à l'université. À ces facteurs, on pourrait ajouter les commémorations des 500 ans de la «découverte» du Brésil, qui ont engendré un vaste mouvement de relecture et de réinterprétation de la formation historique de la nation.

\section{Littérature de jeunesse amérindienne}

Néanmoins, ce constat peut être nuancé si l'on prend en considération un domaine précis, celui de la littérature de jeunesse. Nombreux sont les écrivains qui revendiquent leurs origines amérindiennes et se consacrent à ce genre. Une partie significative de ces ouvrages a été éditée dans le cadre de programmes éducatifs de soutien à la production de matériel pédagogique sur la diversité ethnique, implantés par le gouvernement brésilien, notamment à partir de 2002. Différemment de l'expérience collective qui caractérise la production des textes cités ci-dessus, il s'agit ici d'auteurs individuels qui écrivent en portugais et visent à pénétrer un marché éditorial en pleine effervescence au Brésil. Selon Lynn Mario T. Menezes de Souza, auteur d'un article clairvoyant sur l'écriture amérindienne au Brésil, ces écrivains envisagent de toucher, en priorité, un 
public allochtone (SOUZA, 2003, p. 135). Leur écriture se greffe sur la riche tradition de la littérature orale amérindienne pour revisiter ses légendes, ses mythes, ses traditions ancestrales. Elle est traversée par l'intention d'informer et de transmettre des connaissances sur les cultures des peuples autochtones.

Parmi les écrivains les plus connus, on pourrait citer les noms de Kaká Werá Jecupé (A terra dos mil povos: história indígena do Brasil contada por um índio, 1998); As fabulosas fábulas de Iaruaretê, 2007); Olívio Jekupé (Iarandu o cão falante, 2002); Yaguarê Yamã (Contos da floresta, 2012; Sehaypóri: o livro sagrado do povo SaterêMawê, 2007); Daniel Munduruku (As serpentes que roubaram a noite e outros mitos, 2001; Histórias de índios, 1996); Eliane Potiguara (Akajutibiró, terra do índio Potiguara, 1994); Graça Graúna (Criaturas de Ñanderu, 2010). Ces ouvrages sont pour la plupart publiés par de petites maisons d'édition dont Peirópolis qui a ouvert une ligne éditoriale intitulée «La voix de l'Indien »s'adressant « aux enfants qui s'initient à la lecture, aux enfants avec autonomie de lecture, aux jeunes et aux adultes» (Catalogue Peirópolis, s/d., p. 67). Depuis la loi 11.645 de 2008, qui a rendu obligatoire l'enseignement des cultures amérindienne et afro-brésilienne au sein du système scolaire brésilien, des maisons d'édition plus importantes ont ouvert leurs portes à ce genre de publication.

Le but premier de cette production littéraire est didactique. Elle vise à préserver le patrimoine culturel autochtone et à le diffuser en direction d'un public plus large. C'est ce qui se dégage des différents témoignages d'écrivains comme ceux de Graça Graúna, métisse d'origine Potiguara, de Daniel Munduruku et de Yaguarê Yamã, d'origine Maraguá (Amazonie) pour qui «A história oral é muito bonita e tem que ser mantida», ce qui justifie, selon lui, l'intérêt de passer de l'oral à l'écrit, car «quando a gente passa a escrever essa história, além de passar toda a tradição mostramos o que existe na nossa $»^{3}$. (CORREIO BRAZILIENSE, 2016) Graça Graúna introduit un point de vue intéressant lorsqu'elle affirme que les récits oraux des sociétés traditionnelles ne visent pas une tranche d'âge spécifique, mais c'est la manière de représenter les mythes, les légendes, les aventures et les coutumes qui éveille l'intérêt des enfants et des adultes. Si le système littéraire brésilien classe ces ouvrages dans le domaine de la littérature de jeunesse, c'est parce qu'il a compris que leur caractère merveilleux et les graphismes singuliers 
des ethnies attirent particulièrement le public jeune. Graça Graúna rappelle également que des auteurs tels que Daniel Munduruku, Yaguarê Yamã, Renê Kithãulu et Olívio Jekupé préfèrent, au contraire, les classer comme «contação de histórias» (histoires racontées). Ce désaccord est un signe de la difficulté à dépasser les barrières du passage du récit traditionnel oral à l'écrit ainsi que celles qui résultent de la méconnaissance de la cosmovision amérindienne par la société nationale.

\section{Fiction et poésie amérindiennes}

Même s'ils sont peu nombreux à aller au-delà de la production de «livres pour la jeunesse», certains auteurs ont osé emprunter le chemin de l'écriture de romans et de poèmes visant leur insertion dans le système littéraire brésilien pour lutter contre l'exclusion matérielle et symbolique dont les Amérindiens sont victimes. C'est le cas de Daniel Munduruku, né à Belém, en 1964, auteur du roman Todas as coisas são pequenas (2008); Graça Graúna, née à São José do Campestre, Rio Grande do Norte, en 1948, auteur de trois recueils de poèmes Canto Mestizo (1999); Tessituras da Terra (2001) et Tear da Palavra (2007) et Eliane Potiguara, née en 1950, à Rio de Janeiro, auteure d'un ouvrage inclassable, Metade cara, metade máscara (2004). Ils sont tous nés en ville, leurs ancêtres Munduruku et Potiguara (dans le cas des deux écrivaines) ayant quitté leurs terres traditionnelles depuis des décennies. Leur projet littéraire est à la fois redevable à l'espace social urbain où ils sont nés, ont grandi et ont été éduqués et à l'imaginaire culturel amérindien qu'ils ont redécouvert pour se le réapproprier dans des textes qui portent les marques de leur identité hybride. L'éducation universitaire au Brésil comme vecteur d'ascension sociale, permettant à l'individu de dépasser les barrières qui l'assignent à un lieu précaire, est aussi un facteur important dans les trajectoires de ces trois écrivains: deux d'entre eux, Daniel Munduruku et Graça Graúna sont devenus docteurs et professeurs d'université. Graça Graúna est aussi critique littéraire avec des travaux consacrés aux littératures et cultures amérindiennes, notamment l'ouvrage Contrapontos da literatura indígena contemporânea no Brasil (2013).

Pour cette génération d'Amérindiens qui écrivent, l'accès à l'éducation est une tactique de lutte: la formation intellectuelle les qualifie pour influer dans la redéfinition 
des rapports entre les Amérindiens et l'État brésilien. Leur projet de création et de lutte politique passe par la maîtrise de connaissances et de formes occidentales de transmission du savoir pour mieux défendre leurs droits et faire connaître l'histoire des différents peuples ainsi que leurs références culturelles singulières ${ }^{4}$.

En plein $\mathrm{XXI}^{\mathrm{e}}$ siècle, la littérature amérindienne en langue portugaise constitue un élément nouveau dans l'univers de ce que l'on appelle au Brésil les littératures des minorités, à l'exemple de «la littérature marginale», issue des périphéries des mégalopoles, de la littérature des Afro-descendants ou de la littérature féminine. En se tournant vers les productions des trois auteurs cités, on observe que le roman de Daniel Munduruku est une sorte de fable morale construite à partir d'une vision dichotomique sur les valeurs sous-jacentes aux sociétés occidentale et amérindienne, auxquelles sont attribuées des caractéristiques stéréotypées. Le discours du narrateur-personnage blanc qui veut transmettre aux Occidentaux une leçon de vie, en faisant l'éloge de vraies valeurs qu'il a découvertes lors de sa cohabitation avec une tribu de l'Amazonie, sonne faux. Sa portée moralisante empêche le lecteur d'adhérer au récit.

L'œuvre d'Eliane Potiguara et les recueils de poèmes de Graça Graúna sont plus complexes dans leur approche des relations entre les deux sociétés dans la mesure où ils ne se limitent pas à la logique binaire des conflits, même s'ils n'évacuent pas les tensions issues de la dynamique des contacts culturels et de l'héritage colonialiste. L'intérêt de la lecture de leurs textes réside, à mon avis, dans l'ampleur de la perspective adoptée: l'écriture est pour ces écrivaines, en priorité, un instrument de lutte en faveur des peuples amérindiens, mais leur pensée envisage globalement la précarité matérielle et symbolique qui condamne aussi bien les Amérindiens que les Afro-descendants, les pauvres de la périphérie et les sansterre que les femmes. L'espace symbolique construit est celui de la confluence des voix qui émergent des lieux précaires et marginalisés et qui produisent des textes hybrides comme les voix qui les portent. C'est le cas du poème de Graça Graúna, «Feitura de Tupã», dédié à Gonçalves Dias, inspiré de son poème «Marabá» (Últimos cantos):

Quando Marabá deixou a tribo

Não foi por querer.

Sendo filha de quem é, enfrentou as duras penas

De ser o que é 


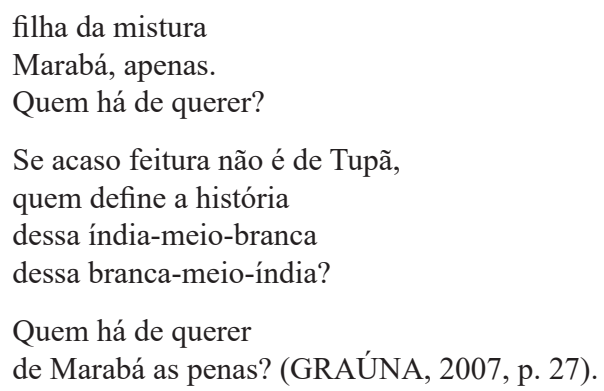

La question de l'hybridisme culturel est soulevée par l'écrivaine lorsqu'elle commente le poème de Gonçalves Dias dans son essai Contrapontos da literatura indígena contemporânea brasileira (2013): «Marabá configura a atualidade do texto de Gonçalves Dias ao retratar a «nossa» condição de filhos da mistura, filhos da erva (na visão indígena), filhos de Eva (na visão ocidental cristã); filhos marginalizados, espoliados, excluídos» (GRAÚNA, 2013, p. 51). L'approche identitaire ambivalente problématise l'identité métisse.

C'est ce qu'on découvre à la lecture de Metade cara, metade máscara d'Eliane Potiguara, texte emblématique de nouvelles formes et thématiques littéraires que la littérature amérindienne naissante inaugure.

\section{Metade cara, metade máscara d'Eliane Potiguara}

Pour examiner la façon dont l'ouvrage surgit dans le système littéraire brésilien, je commencerai par des considérations qui concernent les éléments de son paratexte, tels que la présentation de la collection et la préface du livre. Publié en 2004, par une grande maison d'édition, la Global Editora de São Paulo, l'ouvrage est malheureusement épuisé et le catalogue de la maison d'édition ne le mentionne même plus.

La présentation de la collection «Visões de índio», dans laquelle le livre s'inscrit, est signée par Daniel Munduruku, qui est peut-être l'écrivain amérindien le plus connu au Brésil par son investissement dans la diffusion des cultures amérindiennes; il est notamment l'auteur du roman Todas as coisas são pequenas et de nombreux titres classés littérature de jeunesse. Ce texte de présentation se propose de «donner la voix aux Indiens en mouvement» et de «faire éclore la pensée, la sensibilité, la façon de vivre des peuples 
indigènes brésiliens» (POTIGUARA, 2004, p. 16). Le titre donné à cette présentation «Visões de ontem, hoje e amanhã : é hora de ler as palavras» annonce la volonté de figurer l'histoire des Amérindiens dans une continuité temporelle, depuis son inscription dans une perspective de longue durée, en passant par l'affirmation de sa contemporanéité et en la projetant dans l'avenir, défi que la production littéraire se chargerait de relever. La préface de Graça Graúna, écrivaine et professeur de l’Universidade Federal de Pernambuco, lui confère une caution littéraire qui contribue à sa reconnaissance. Elle souligne le caractère pionnier de l'œuvre et son importance pour pénétrer dans l'histoire et l'univers culturel des peuples autochtones, sans oublier de signaler le dialogue tendu qu'elle inaugure avec le modèle social hégémonique de la société nationale brésilienne.

Metade cara, metade máscara rassemble des textes pionniers écrits par l'auteure depuis les années 1970. Premier ouvrage littéraire amérindien écrit en portugais à acquérir une certaine répercussion dans le milieu académique et littéraire, il constitue un des rares exemples de publication d'un auteur qui revendique son identité amérindienne, porteur d'un projet de réappropriation historique et culturelle. La force et la nouveauté de cet ouvrage de référence se trouvent dans la connexion qu'il établit entre la contemporanéité et l'ancestralité pour représenter la reconstruction de l'identité amérindienne, comme l'a bien observé Rejane S. Gelhen dans la lecture perspicace qu'elle lui a consacrée (GEHLEN, 2011, p.86). Il ne faut néanmoins pas mépriser la trajectoire sociale de l'auteure, biologiquement et culturellement métissée, militante amérindienne avant la lettre, engagée depuis les années 1970 dans la lutte contre la violence envers les femmes amérindiennes, membre fondateur de l'association GRUMIN - Grupo Mulher-Educação Indígena.

D’origine amérindienne, l'auteure a adopté le nom de son peuple Potiguara qui occupait les territoires correspondant aujourd'hui, en partie, aux États du Nordeste du Brésil, depuis le littoral du Maranhão jusqu'à l'État de la Paraíba; de nos jours, leur territoire se concentre entre les états du Rio Grande do Norte et de Paraíba (DANTAS et al., 2008, p. 439). Sa famille, victime des violences des propriétaires terriens, dépossédée de sa terre tribale dans la décennie de 1920, a d'abord migré vers Pernambuco avant de poursuivre son chemin vers la périphérie de Rio de Janeiro où l'écrivaine est née en 1950. Le noyau familial de l'écrivaine, devenue orpheline dans sa première enfance, 
n'est composé que de femmes: la grand-mère, la mère, une tante et la sœur de l'auteure. Dans l'univers de ces femmes, les hommes sont les grands absents, ayant été assassinés comme son arrière-grand-père, victime de la lutte qu'il menait pour la préservation des territoires traditionnels des Potiguara. Sa grand-mère, victime d'un viol, a enfanté à l'âge de douze ans.

Depuis son plus jeune âge, Eliane Potiguara a été victime de préjugés et de violences. La prise de conscience de sa condition subalterne l'a amenée à s'engager dans la lutte pour la structuration du mouvement indien à l'échelle nationale et internationale, comme en témoigne, entre autres, sa participation aux discussions pour l'élaboration de la Déclaration des Nations Unies pour les droits des peuples autochtones. Elle a pu, bien que tardivement, avoir accès à l'éducation, devenir institutrice, fréquenter le milieu culturel de Rio de Janeiro et publier des ouvrages ${ }^{6}$ Dans Metade cara, metade máscara, elle évoque son parcours de vie, s'interroge sur les relations entre autochtones et nationaux tout en dénonçant la violence coloniale et néocoloniale envers les Indiens, sans renoncer pour autant à y faire figurer un projet de cohabitation.

Son expérience de vie en tant qu'amérindienne et femme, acteur social doublement discriminé, nourrit une expérience d'écriture qu'elle envisage comme une «politique de l'existence», à la fois dénonciation des violences contre les Autochtones et forme de thérapie: «Depois da angústia e do desespero, o ato de criação é o começo da cura!»» (POTIGUARA, 2004, p. 58). Cette voix féminine instaure son propre espace d'énonciation en problématisant son identité amérindienne et son appartenance à la société brésilienne. Elle évoque la dissolution brutale de l'espace habité amérindien (Harel, 2007, p. 87) depuis l'arrivée des colonisateurs, qui a contraint nombre de peuples autochtones à migrer; elle dénonce les injustices qu'ils ont subies, avec la dépossession de leurs terres et les violences physiques, morales et symboliques; elle envisage la réappropriation de la mémoire et de l'histoire amérindienne, tout en inscrivant ces peuples dans l'avenir de la nation brésilienne:

Nós, povos indígenas,

Queremos brilhar no cenário da História

Resgatar nossa memória

E ver os frutos de nosso país, sendo divididos 


\author{
Radicalmente \\ Entre milhares de confinés s e «desplazados» \\ Como nós ${ }^{8}$ \\ (POTIGUARA, 2004, « Identidade indígena », p. 104).
}

Eliane Potiguara explore également l'archétype féminin, l'image de la femme qui engendre la vie tout comme «la mère-terre»: «É com a mulher que o homem aprende. É com a mãe-terra, é com o ventre vulcânico revolucionário, guerreiro, combativo que trará a transformação do ser humano» (POTIGUARA, 2004, p. 97). Elle puise ainsi dans le mythe du renouvellement pour légitimer sa parole de femme amérindienne en lui attribuant la force primordiale de transformation qui alimente ses actes et son discours et crée le personnage Cunhataí, alter ego de l'écrivaine, dont elle souligne le rôle dans un de ses fragments autoréférentiels:

\begin{abstract}
Na realidade, a simbologia de Cunhataí demonstra o compromisso que ela tem com todas as mulheres indígenas do Brasil. Sua dor, sua insatisfação e consciência de mulher é a mesma trazida pelas mulheres guerreiras dos tempos atuais, que ora se organizam ${ }^{9}$ (POTIGUARA, 2004, p. 70).
\end{abstract}

L'ouvrage présente une composition complexe qui se caractérise par la compilation de plusieurs textes, écrits au long d'une trentaine d'années entre 1975 et 2003, répondant au principe de la polytextualité, caractérisé par «sa multiplicité textuelle, par son statut d'agrégat [qui] met ipso facto l'idée du livre en péril: sa cohérence, sa fluidité sont compromises» (AUDET, 2016, p. 136). La puissance de la voix féminine démultipliée contribue à l'éclatement de l'unité énonciative, d'où la multiplicité de voix auctoriales et de genres: autofiction, autobiographie, témoignage, chronique, poésie, fiction poétique qui évoque l'univers symbolique des mythes. L'articulation du vécu à l'histoire et à la subjectivité poétique se manifeste dans l'histoire revisitée qui est aussi bien celle des peuples amérindiens que celle de l'organisation politique du mouvement indien au Brésil, auxquelles s'entrelace le parcours de vie de l'écrivaine. Dès le titre, Metade cara, metade máscara, le recueil suggère les métamorphoses de l'identité en rapport avec la démultiplication de la voix qui peut parler au nom de l'individu ou d'une collectivité (femmes amérindiennes, Amérindiens, Brésiliens opprimés) pour mettre au jour, sous 
différents aspects, les conséquences dramatiques de la colonisation pour les peuples et les cultures amérindiennes. Selon certains mythes amazoniens, le masque est doté d'un pouvoir transformationnel dont celui de la guérison en rapport avec la fonction thérapeutique que l'auteur attribue à la création littéraire.

Il s'agit d'un recueil structuré autour de sept chapitres qui dévoilent les empreintes tragiques de l'histoire des Amérindiens à partir d'un point de vue prédominant qui exprime la souffrance féminine. La multiplicité de textes impliqués introduit la modulation de la voix dans l'écriture et les nuances dans la perception de l'histoire. Ainsi, la chronique qui ouvre le recueil rappelle, dans un récit à la troisième personne, les violences subies par les Amérindiens depuis l'arrivée des colonisateurs pour, dans un deuxième mouvement, introduire un texte autofictionnel consacré aux agressions endurées par les Potiguara et la famille des ancêtres de l'auteure dans les années 1920. Dans un autre chapitre, le sujet de l'énonciation assumera la narration de cette même histoire, en première personne directe, dans le cadre d'un récit autobiographique. Ce choix narratif rapproche la dimension ontologique du récit de vie du processus historique, car ce qu'il met en relief, c'est le destin commun à de nombreuses femmes amérindiennes; les auto-histoires (GRAÚNA, 2013, p. 131) collectives et s s'entremêlent.

La contribution effective d'Eliane Potiguara à l'organisation du mouvement indien au Brésil, en particulier dans la période initiale et difficile de sa constitution, à partir de 1974, sous la dictature militaire, lui permet d'écrire des chroniques consacrées à cette histoire et de rappeler les principales revendications de ce mouvement. Ces textes portent les marques de l'engagement de l'auteur: le discours militant dénonce les injustices et les violences subies par les peuples indigènes au Brésil et se positionne en faveur de la défense de leurs droits:

A demarcação das terras indígenas nunca foi uma prioridade governamental. Uma política que garantisse e respeitasse os povos indígenas como unidades sociopolíticas e culturais distintas deveria ser uma prioridade como respeito histórico. Nunca se realizou, na prática, uma política voltada aos interesses e projetos econômicos de auto-sustentação propostos pelos indígenas, baseados em sua biodiversidade com segurança para a saúde, educação, agricultura e direitos humanos, considerando sua cultura diferenciada(POTIGUARA, 2004, p. 44). 
L'articulation de la question indienne à la problématique identitaire émerge dans des textes poétiques qui insèrent la perspective de la subjectivité féminine, comme on peut l'observer dans des poèmes qui interrogent le processus complexe de construction identitaire de la femme amérindienne au sein de la société brésilienne («Que faço com minha cara de india?», «Brasil»); dénoncent les massacres et les violences subies par les femmes amérindiennes («Quem são vocês que podem violentar/A filha da terra/E retalhar suas entranhas $?^{11} »$, «Invasão»); répercutent les revendications des Peuples Premiers («Porque só pedimos terra e paz/Pra nossas pobres - essas ricas crianças», «Oração pela libertação dos povos indígenas» ${ }^{12}$ ); s'apitoient sur la condition précaire des Amérindiens à laquelle le sujet lyrique s’identifie («Ah !... Não sei mais continuar esses cânticos/Porque a mim tudo foi roubado», «Migração indígena»); projettent l'élargissement des horizons féminins («Mãe, fêmea, amante sécula/Mas com seus direitos de mulher», «Homem», (POTIGUARA, 2004, p. 133 $33^{13}$ ).

La fiction poétique qui évoque l'histoire d'amour entre Jurupiranga et Cunhataí introduit un plan allégorique qui illustre, poétiquement, le parcours tragique de la diaspora amérindienne et fait écho à l'itinéraire de l'écrivaine:

Jurupiranga e Cunhataí são dois personagens do texto Ato de amor entre povos das próximas páginas, que sobreviveram à colonização e poeticamente vão nos contar suas dores, lutas e conquistas. Esses personagens são atemporais e sem locais específicos de origem ${ }^{14}$ (POTIGUARA, 2004, p. 30).

L'histoire «atemporelle du couple Jurupiranga et Cunhataí, emblème de la famille indigène» (FIGUEIREDO, 2013, p. 153) va ponctuer la traversée des différents textes, tissant des liens de continuité entre eux, selon une dialectique autonomie/intégration propre aux pratiques polytextuelles. Ces répétitions relient formellement et temporellement les différents niveaux de la diégèse et évoquent l'atmosphère des récits oraux traditionnels. La récurrence de ces personnages correspond à une «stratégie compensatoire d'ordre thématique» qui, comme le souligne René Audet, «participe à une certaine forme de cohésion de l'ensemble» (AUDET, 2016, p. 136). Ils exercent également la fonction d'agencement de temporalités différentes. 
L'amour de ce couple symbolique amérindien est soumis aux épreuves de la séparation en conséquence des migrations forcées, des ravages des guerres que tous deux doivent affronter, au temps des colonisateurs et dans le présent. L'avenir, annoncé par la fiction poétique, est celui où Éros vaincra Thanatos, celui de la victoire des peuples amérindiens - intitulé du dernier chapitre de l’ouvrage («Vitória dos povos») - qui passe par la récupération de la mémoire et des références culturelles amérindiennes signifiée par le retour de Jurupiranga au village et par ses retrouvailles avec Cunhataí. Pour clore l'ouvrage, l'auteure reprend les fragments du poème «Ato de amor aos povos», écrit en 1978, en hommage aux Peuples Premiers de l'Amérique latine, dans lequel la voix de Cunhataí invite son bien-aimé aux jouissances de l'amour et de la vie:

- Desperta JURUPIRANGA!

Vem me ver que hoje acordei suada.

Benzo,

Com o sumo de minha rosa aberta, enamorada, As manhãs de delírio, completamente cansada. ${ }^{15}$ (POTIGUARA, «Ato de amor aos povos», 2004, p. 136).

L'irruption de la parole amoureuse dans le plan allégorique et poétique de l'œuvre est porteuse d'espoir. C'est un des moyens auxquels la fonction libératrice de l'écriture d'Eliane Potiguara a recours pour reconfigurer l'imaginaire sur les Amérindiens:

\footnotetext{
Nosso ancestral dizia: Temos vida longa!/[...] E transformaremos os sexos indígenas/em órgãos produtores de lindos bebês guerreiros do futuro/e não passaremos mais fome/ ome de alma, fome de terra, fome de mata/fome de História/e não nos suicidaremos/a cada século, a cada era, a cada minuto/Nós somos o primeiro mundo! ${ }^{16}$ (POTIGUARA, «Identidade indígena», 2004, p. 104).
}

Le discours littéraire s'engage dans un combat qui vise à renverser les idées reçues et à engendrer un retournement de l'imaginaire hégémonique sur les Amérindiens qui les refoule et les fige dans un passé historique, quand il ne les cantonne pas dans le seul univers mythique. Le parti pris de son écriture est celui d'inscrire les peuples et les cultures autochtones dans le temps de la longue durée, en opposition aux forces sociales qui travaillent à l'effacement de leur mémoire et à la dépossession de leur terre. Cette voix amérindienne fait irruption dans la littérature brésilienne en marquant sa dissociation 
de l'image que la société nationale lui renvoie, par un travail de renversement de sens: «Nous sommes le premier monde», c’est le refrain du poème «Identidade indígena», écrit en 1975, en hommage à ses grands-parents. Ce poème est le plus ancien de l'ouvrage et, comme le signale Graça Graúna dans sa préface, c'est le premier poème écrit en portugais par une femme amérindienne «à avoir acquis une répercussion nationale et à avoir driblé la censure et le régime militaire» (POTIGUARA, 2004, p. 18). Cette voix amérindienne cherche à déchirer le monopole de la représentation que les élites au pouvoir ont construit comme légitime, au fil du temps. Selon Simon Harel «les sujets exclus doivent composer avec la violence d'un monde qui tente de les expulser du domaine même de la représentation» (HAREL, 2007, p. 90). On peut interpréter l'appropriation de l'écriture littéraire par les Amérindiens comme une tactique de «braconnage», une façon de survivre dans un territoire qui leur est hostile, une manifestation de résistance face au processus d'extermination mis en place depuis des siècles.

Cen'est en fait qu'au XXI ${ }^{\mathrm{e}}$ siècle que surgissent des ouvrages littéraires amérindiens, écrits en portugais, cherchant à se frayer un passage dans le champ de la production littéraire brésilienne pour affirmer leur spécificité ethnique et leur projet d'émancipation vis-à-vis du monopole de la représentation littéraire visant le dépassement d'un imaginaire national «calqué sur le 'syndrome de l'extinction' - expression employée par Márcio» Santilli (2000, p. 21) pour caractériser un type de représentation qui conçoit les Indiens «comme des êtres du passé issus de la littérature indianiste», et «sans espérances de futur» (OLIVIERI-GODET, 2015, p. 8). Eliane Potiguara y joue un rôle prépondérant avec un ouvrage original, aussi bien par sa composition polytextuelle que par la démultiplication de la voix féminine, ce qui lui permet d'évoquer, sous différents aspects, la problématique des Amérindiens au Brésil. Elle met en valeur les tactiques de résistance des femmes amérindiennes et afro-descendantes pour faire face aux néo-colonisateurs; elle ravive la flamme des savoirs ancestraux et de la cosmologie des Peuples Premiers; elle revisite l'histoire amérindienne en y incluant celle de sa résistance et de son organisation politique; elle rappelle que la notion de territoire ne se limite pas à l'espace géographique, comme le démontre le rapport singulier que les Amérindiens entretiennent avec le leur: 
Um território não é apenas um pedaço ou vastidão de terras. Um território traz marcas de séculos, de cultura, de tradições. É um espaço verdadeiramente ético, não é apenas um espaço físico como muitos políticos querem impor. Território é quase sinônimo de ética e dignidade. Território é vida, é biodiversidade, é um conjunto de elementos que compõem e legitimam a existência indígena. Território é cosmologia que passa inclusive pela ancestralidade ${ }^{17}$ (POTIGUARA, 2004, p. 105).

C'est par l'écriture, qui lui rend le rôle de sujet de sa propre histoire, qu'Eliane Potiguara fait l'expérience de «l'habitabilité», ce qui veut dire, selon Simon Harel, «le fait d'être sujet dans un espace circonscrit» (HAREL, 2007, p. 17). Pour cette amérindienne desaldeada, comme pour tant d'autres Amérindiens urbains, redevenir Indien signifie mener un combat en faveur de la reconfiguration des rapports à l'intérieur même de la société brésilienne: pour l'écrivaine Eliane Potiguara, il est question de s'emparer de la mémoire de son patrimoine ancestral pour créer, par sa parole libératrice, son propre habitat:

Eu não tenho minha aldeia/Minha aldeia é minha casa espiritual/ Deixada pelos meus pais e avós/A maior herança indígena./Essa casa espiritual/É onde vivo desde a mais tenra idade ${ }^{18}$ (POTIGUARA, 2004, «Eu não tenho minha aldeia», p. 130).

Cette «maison spirituelle» le sujet poétique l'a héritée de ses ancêtres. Reconstruire la «nation indienne» est une affaire de réappropriation mémorielle, de prise de conscience politique et culturelle que la dynamique temporelle instaurée par le texte permet d'accomplir en faisant cohabiter mythes, ancestralité et contemporanéité:

Cunhataí viaja pelo tempo e espaço, e depois de seguir trilhas e sofrer todas as dores que uma mulher pode sofrer ela para, senta-se e reclina a cabeça ao chão. Absorta nos passos de um formigueiro, ouve vozes intercaladas e no meio delas escuta a voz ancestral ${ }^{19}$ (POTIGUARA, 2004, p. 79).

Néanmoins, la reconstruction du lieu habitable entreprise par le sujet n'est pas envisagée comme un repli identitaire. Elle est réinvestie d'une nouvelle symbolique qui célèbre l'émergence des singularités qui peuvent cohabiter et/ou évoluer vers des phénomènes d'hybridation culturelle. C'est ce qui ressort du rêve visionnaire de Jurupitanga dans lequel la renaissance amérindienne compose avec des éléments de la société brésilienne: 
Os brancos diziam que estavam reconhecendo a dívida histórica que aquele país tinha para com os povos tradicionais e por isso tinham decidido - politicamente - aceitar, pacificamente, as demandas que os povos apresentavam para o exercício dos direitos indígenas. [...] Num sobressalto e num piscar de olhos, vislumbrou a universidade indígena lotada de jovens, futuros antropólogos, cientistas, historiadores, jornalistas, juristas, contadores de sua própria história. Viu bibliotecas inteiras recheadas de livros escritos pelos próprios indígenas, viu uma qualidade de vida nunca vista em toda sua vida. Mulheres indígenas eram respeitadas quando passavam nas cidadelas ao fazerem suas compras ou quando necessitavam de recursos médicos, educacionais, jurídicos. Os velhos eram venerados por todos ${ }^{20}$ (POTIGUARA, 2004, p. 129).

L'allégorie du retour de Jurupiranga à son village dépasse la dichotomie Amérindien/ société nationale par la figuration d'un espace interstitiel dans lequel les aspects traditionnels des cultures amérindiennes cohabitent avec des éléments de la culture brésilienne dans un processus de contamination réciproque. Cette image peut nous paraître d'un romantisme un peu naïf, tant elle détone avec la réalité violente dénoncée par l'ouvrage, à laquelle les Amérindiens doivent faire face dans ce $\mathrm{XXI}^{\mathrm{e}}$ siècle. Comme le signale Ailton Krenak, «les Indiens n'ont aucun lieu pour vivre dans ce pays. Ils sont obligés de faire exister cet endroit au jour le jour» (KRENAK, 2015, p. 19). C'est cette conscience de «braconniers» qui, selon Harel, amène les Amérindiens à composer «avec le territoire de l'autre, puisqu'ils ne peuvent y échapper» (HAREL, 2007, p. 97). Car, «la frontière n’a [...] jamais cessé d'avancer dans les territoires indiens, créant des conditions d'échanges et pas seulement des frictions», comme le signale Jean-Philippe Belleau (2014, p. 14).

Cette tension thématique est renforcée par la stratégie discursive de l'œuvre au niveau même de sa structure, comme j'ai essayé de le montrer. Ainsi, le plan allégorique, porteur d'un message d'espoir eu égard à la renaissance des cultures amérindiennes et à leur cohabitation avec la société brésilienne, exprime le plus profond désir d'une voix amérindienne féminine qui n'hésite pas à exposer les injustices séculaires commises contre les peuples autochtones.

Eliane Potiguara fait de l'écriture une arme tactique pour se reconstruire en tant que femme amérindienne, affronter la réalité et projeter un avenir pour les Amérindiens au Brésil: «Minha vida é permeada pela questão ética, racial e de gênero» ${ }^{21}$, affirme-t- 
elle dans un témoignage (FOUILLEUL, 2016, p. 75). L'émergence de nouvelles voix amérindienne et afro-brésilienne dans la littérature brésilienne est marquée, comme le remarque avec raison Eurídice Figueiredo (2013, p. 154), par l'entrecroisement de l'ethnicité et du genre engendrant de nouvelles versions de la mémoire collective. Ces voix émergentes révèlent un parcours différencié de leur propre histoire et de leur mémoire. Elles sont engagées dans un processus de production de discours qui se superpose au silence de plusieurs siècles qui s'est abattu sur leurs histoires afin de guérir l'amnésie de la société brésilienne et d'ouvrir les portes à des mémoires multiples et différenciées de la mémoire officielle.

Ainsi, le plus grand intérêt de l'ouvrage d'Eliane Potiguara réside probablement dans sa participation originale au processus décolonisateur des lettres brésiliennes qui consiste à assumer le rôle de porte-parole des revendications et des droits des Amérindiens, sans se retrancher derrière des frontières identitaires essentialistes, comme c'est souvent le cas dans la production de la littérature dite des minorités. Son regard hybride de descendante d'Indiens migrants se pose sur les hétérogénéités qui constituent l'espace national conflictuel, mais refuse de projeter le dépassement des conflits par l'uniformisation des différences. L'inclusion citoyenne de l'Amérindien ne peut se faire que par le respect de ses singularités. Les deux grands mouvements de la dynamique spatiale de l'œuvre sont les piliers de cette représentation: l'un, proche du plan du réel, dénonce le processus de dépossession des territoires et récupère la mémoire de l'exclusion dont les Amérindiens ont été victimes tout au long de l'histoire; l'autre, sur le terrain du projet utopique de l'œuvre, projette un espace perméable à la cohabitation et aux échanges dans lequel évoluent des identités composites, ce qui conforte l'avis de Graça Graúna selon lequel «la littérature amérindienne contemporaine est un lieu utopique (de survie)» (GRÁUNA, 2013, p. 15). L'émergence de la voix féminine d'Eliane Potiguara ouvre ainsi un chemin vers l'inclusion de l'écriture amérindienne dans le système littéraire brésilien en déchirant le monopole de la représentation des Peuples Premiers par des écrivains Non-Indiens.

L'émergence des voix amérindiennes dévoile un mode spécifique d'être au monde, mais rend également problématique la revendication de cette spécificité ethnique en littérature. Elle nous invite à réfléchir sur les conséquences que la recherche de cette 
visibilité ethnique en littérature peut avoir sur l'enfermement dans des thématiques qui correspondent à l'horizon d'attente du lecteur. Comment s'affranchir de cette spécificité ethnique et s'ouvrir aux pratiques illimitées du champ esthétique? Le jour où cela arrivera, cela signifiera le dépassement d'une étape, nécessaire, mais contraignante, de revendication et d'affirmation identitaire, caractéristique des productions littéraires qui émergent comme porte-paroles d'une communauté.

\section{Références}

ALMEIDA, Maria Inês de. Entrevista, 2011.

Accessible sur le site http://txaihunikuin.blogspot.fr/2011/05/entrevista-da-professoramaria-ines-de.html

ALMEIDA, Maria Inês de. Desocidentada. Experiência literária em terra indígena. Belo Horizonte: Editora UFMG, 2009.

AUDET, René. L'autorité de la fiction dans des œuvres polytextuelles. In: BOUJOU, Emmanuel. L'autorité en littérature. Rennes : PUR, 2016, p. 133-139.

BELLEAU, Jean Philippe. Le mouvement indien au Brésil. Du village aux organisations. Rennes : PUR, 2014.

BUENO, E. Brasil: uma história. São Paulo: Ática, 2003, p. 18-19.

CINTA LARGA, Pichuvy. Org. Ana Leonel de Queiroz, Ivete Lara Camargos Walty e Leda Lima Leonel. Mantére ma Kwe tïnhim - Histórias de maloca antigamente. Belo Horizonte: SEGRAC-CIMI,1988. Accessible sur le site emad.fflch.usp.br/sites/lemad. fflch.usp.br/files/HISTÓRIAS DE MALOCA ANTIGAMENTE.pdf

CUNHA, Rebelise da; SANTOS, Eloína. Apresentação: À procura de novos paradigmas: estudos indígenas no Canadá e nas Américas. Interfaces Brasil/Canadá, Vol 16, n. 3, 2016. cessible sur le site: https://periodicos.ufpel.edu.br/ojs2/index.php/ interfaces/article/view/10243/6754

DANTAS, Beatriz G.; SAMPAIO, José Augusto L; CARVALHO, Maria Rosário G. Os povos indígenas no Nordeste brasileiro. Um esboço histórico. In: CUNHA, Manuela Carneiro de. História dos índios no Brasil. São Paulo: Companhia das Letras, 2008, p. 431-456.

FIGUEIREDO, Eurídice. Políticas e poéticas da memória: gênero e etnicidade (Conceição Evaristo e Eliane Potiguara). In: Mulheres ao espelho: autobiografia, fiç̧ão, autoficção. Rio de Janeiro: EDUERJ, 2013, p. 149-167.

FOUILLEUL, Anaís. Les voix des femmes amérindiennes dans les littératures des Amériques. Mémoire soutenu le 20 septembre 2016, sous la direction de Rita OlivieriGodet, dans le cadre du Master "Les Amériques" de Université Rennes 2. Accessible 
sur le site http://www.mulherespaz.org.br/acoes-das-mulheres-da-paz/evento-jovens-nacampanha-nacional-mulheres-pela-paz/

GEHLEN, Rejane Seitenfuss. Identidade de Eliane: a face Potiguara, a máscara indígena e o eco de vozes silenciadas. Boitatá - Revista do GT de Literatura Oral e Popular da ANPOLL. Londrina, n. 12, p. 81-103, jul-dez 2011.

GENETTE, Gérard. Seuils. Paris: Seuil, 1987.

GRAÚNA, Graça. Tear da palavra. Belo Horizonte: S.N., 2007.

GRAÚNA, Graça. Criaturas de Ñanderu. São Paulo: Ed. Manole, 2010.

GRAÚNA, Graça. Contrapontos da literatura indígena contemporânea brasileira. Belo Horizonte: Maza Edições, 2013.

HAREL, Simon. Espaces en perdition. Tome I. Les lieux précaires de la vie quotidienne. Québec: PUL, 2007.

HAREL, Simon. Espaces en perdition. Tome II. Humanités jetables. Québec: PUL, 2007.

JECUPÉ, Kaká Werá. A terra dos mil povos: uma história do Brasil contada por um Índio. São Paulo: Peirópolis, 1998.

LIMA, Antônio Carlos de Souza e Barroso, Maria Macedo (Org.). Povos indígenas e universidade no Brasil: contextos e perspectivas, 2004-2008. Rio de Janeiro : E-papers, 2013.

OLIVIERI-GODET, Rita. L'altérité amérindienne dans la fiction contemporaine des Amériques. Brésil, Argentine, Québec. Québec : Presses de 1'Université Laval, 2015.

OLIVIERI-GODET, Rita. Figurations des espaces amérindiens dans les littératures du Brésil et du Québec. Expériences et écritures de l'espace au Québec et au Brésil, Rita OLIVIERI-GODET, Licia SOARES DE SOUZA, Brigitte THERION (Dir.). Interfaces Brasil/Canadá, Vol 15, n² 2, décembre 2015. https://periodicos.ufpel.edu.br/ojs2/index. php/interfaces/article/view/7282/5101.

POTIGUARA, Eliane. Metade cara, metade máscara. São Paulo: Global, 2004.

. Eliane. Akajutibiró: terra do índio Potiguara. Unesco, 1994.

. Eliane. A terra é a mãe do índio. Rio de Janeiro: Grumin, 1989.

. Eliane. Interview. Accessible sur le site http://www.mulherespaz.org.br/acoesdas-mulheres-da-paz/evento-jovens-na-campanha-nacional-mulheres-pela-paz/

RIBEIRO, Darcy. O povo brasileiro: a formação e o sentido do Brasil. [S.1.]: Companhia das Letras, 1995, p. 470. ISBN: 9788571644519.

SANTILLI, Márcio. Os brasileiros e os índios. São Paulo: Senac, 2000.

SANTOS, Eloína Prati dos. O refuncionamento da narrativa na ficção ameríndia Contemporânea do Brasil, Estados Unidos e Canadá. In: . E.P. (Org.). Perspectivas da literatura ameríndia no Brasil, Estados Unidos e Canadá. Feira de Santana: 
Universidade Estadual de Feira de Santana, 2003, p. 43-58. Accessible sur le site http:// nec.furg.br/index.php/acervo.html

SOUZA, Lynn Mario T. Menezes. Que história é essa? A escrita indígena no Brasil. In: SANTOS, Eloína Prati dos (Org.). Perspectivas da literatura ameríndia no Brasil, Estados Unidos e Canadá. Feira de Santana: Universidade estadual de Feira de Santana, 2003, p.125-137. Accessible sur le site http://nec.furg.br/index.php/acervo.html

THERY, Hervé. Le Brésil pays émergé. Paris: Armand Colin, 2014.

WALTER, Roland. Prefácio. In: GRAÚNA, Graça. Contrapontos da literatura indígena contemporânea brasileira. Belo Horizonte: Maza Edições, 2013, p. 9-13.

WALTY, Ivete Lara Camargos. Narrativa e imaginário social: uma leitura das histórias de maloca antigamente de Pichuvy Cinta Larga. São Paulo: USP, 1991, 237 p. (Tese de Doutorado).

WALTY, Ivete Lara Camargos. O intelectual e as minorias. Accessible sur le site http:// lasa.international.pitt.edu/Lasa2000/Walty.PDF, consulté le 28/03/16.

YAMÃ, Yaguarê. Literatura indígena: da oralidade ao papel. Correio Braziliense, 3, Brasília, segunda-feira, 29 de fevereiro de 2016. Accessible sur le site ggrauna.blogspot. com/ terça-feira, 1 de março de 2016.

\section{Notes}

1 ERIMIT - Université Rennes 2, Institut Universitaire de France, Alta Bretanha, França. rgodet@9online.fr.

2 Les citations de poèmes et extraits des œuvres en portugais on été traduites par Brigitte Thiérion: «deviennent des vecteurs d'indigénisation de la politique nationale plutôt que de 'brésilianisation' d'Indiens, et qui se réapproprient simultanément leur indianité par le biais intellectuel et existentiel».

3 «les récits de la tradition orale sont très beaux et doivent être préservés» (...) «non seulement on transmet la tradition mais on fait connaître notre culture» (YAMÃ, 2016).

${ }^{4}$ Rubelise da Cunha et Eloina Santos ont organisé le dossier précédente de le reveu Interfaces (v. 16, no. 3, 2016), «En quête de nouveaux paradigmes: études indigènes au Canada et dans les Amériques», de nature comparative entre les écrivains autochtones et non-autochtones au Brésil et au Canada.

5 «Quand Marabá quitta la tribu/Ce fut contre son gré./Étant la fille de qui elle est./Elle souffrit durement/ d'être fille du mélange/Marabá, à peine./Qui aimerait?/Et si par hasard ce n'est pas l'œuvre de Tupã/qui trace l'Histoire/de cette Indienne-à-demi-blanche/de cette Blanche-à-demi-indienne?/Qui aimerait/Marabá et ses peines?»

${ }^{6}$ A terra é mãe do índio (1989); Akajutibiró, terra do indio Potiguara (1994).

7 «Après l'angoisse et le désespoir, l'acte de création est le commencement de la guérison.»

8 «Nous, peuples indigènes,/Nous voulons briller sur la scène de l'Histoire/Restaurer notre mémoire/et voir les fruits de notre pays, être divisés/Radicalement/entre des milliers d'Indiens confinés dans les réserves et “ déplacés'/Comme nous.»

9 «En réalité, le symbolisme de Cunhataí démontre son engagement aux côtés de toutes les femmes indigènes du Brésil. Leur douleur, leur insatisfaction, et sa conscience de femme est la même que celle des guerrières des temps modernes, qui s'organisent aujourd'hui.»

10 «La démarcation des terres indigènes n'a jamais été une priorité gouvernementale. Faire une politique qui protège et respecte les peuples indigènes comme entités sociopolitiques et culturelles distinctes devrait être une priorité au nom du respect historique qui leur est dû. Dans les faits, il n'y a jamais eu aucune politique qui prenne en compte leur différence culturelle et qui encourage les projets économiques d'autosuffisance proposés par les Indigènes et basés sur leur biodiversité pour garantir la santé, l'éducation, l'agriculture et les droits humains.» 
${ }_{11}$ «Qui êtes-vous, vous qui pouvez violer/La fille de la terre/ Et déchirer ses entrailles?» «Invasion».

12 «Parce que nous ne demandons que la terre et la paix/ Pour nos pauvres - ces beaux enfants.» («Prière pour la libération des peuples indigènes»).

${ }^{13}$ («Ah!... Je ne sais continuer ces cantiques/Parce qu'on m'a tout volé.» «Migration Indigène»; («Mère, femelle, amante séculière/Mais avec des droits de femme.» «Homme»).

14 «Jurupiranga et Cunhataí sont deux des personnages du texte Ato de Amor entre povos des prochaines pages. Ils ont survécu à la colonisation et vont nous raconter poétiquement leurs douleurs, leurs luttes et leurs conquêtes. Ces personnages sont intemporels et ne sont originaires d'aucuns lieux en particulier.»

15 «Réveille-toi JURUPIRANGA!/Viens, aujourd'hui je me suis éveillée en sueur./Amoureuse, languide, je bénis,/De ce suc qui perle de ma rose entr'ouverte,/Les matins de délire.»

16 «Notre ancêtre disait: Notre vie est longue!/[...] Et nous transformerons les sexes indigènes/en organes producteurs de beaux bébés, les guerriers du futurs/et nous n'aurons plus faim/faim d'âme, faim de terre, faim de forêt/faim d'Histoire/et nous ne nous suiciderons plus/Chaque siècle, chaque ère, chaque minute/Nous sommes le premier monde!»

17 «Un territoire ce n'est pas seulement un morceau ou une étendue de terres. Un territoire porte l'empreinte des siècles, de la culture, des traditions. C'est un espace véritablement éthique, ce n'est pas seulement un espace physique, comme de nombreux politiques veulent le faire croire. Le territoire est quasiment synonyme d'éthique et de dignité. Le territoire c'est la vie, la biodiversité, c'est un ensemble d'éléments qui composent et légitiment l'existence indigène. Le territoire c'est la cosmologie qui passe également par l'ancestralité.» 18 «Je n'ai pas mon village/Mon village c'est ma maison spirituelle/Celle que m'ont léguée mes parents et mes grands-parents/Le plus grand héritage indigène./Cette maison spirituelle/C'est là où je vis depuis ma plus tendre enfance.»

19 «Cunhataí voyage dans le temps et l’espace, et après avoir suivi les pistes et enduré toutes les douleurs qu'une femme peut endurer elle s'arrête, s'assoit et incline la tête vers le sol. Absorbée sur les traces d'une fourmilière, elle entend des voix entremêlées et parmi elles, la voix ancestrale.»

20 «Les Blancs disaient qu'ils reconnaissaient la dette historique que ce pays avait envers les peuples traditionnels et c'est pourquoi ils avaient décidé - politiquement - d'accepter, pacifiquement, les demandes que les peuples présentaient dans l'exercice de leurs droits indigènes. [...] Sursautant, il aperçut en un clin d'œil l'université indigène remplie de jeunes, futurs anthropologues, scientifiques, historiens, journalistes, juristes, conteurs de sa propre histoire. Il vit des bibliothèques entières pleines de livres écrits par les Indigènes euxmêmes, il nota une qualité de vie qu'il n'avait encore jamais vue de toute son existence. Les femmes indigènes étaient respectées lorsqu'elles passaient dans les citadelles pour y faire leurs achats ou quand elles avaient besoin de ressources médicales, éducatives, juridiques. Les vieux étaient respectés de tous.»

${ }^{21}$ «Ma vie est traversée par la question éthique, raciale et de genre». (FOUILLEUL, 2016, p. 75). 